\title{
NUMERICAL DETERMINATION OF CONCRETE CRACK WIDTH FOR CORROSION-AFFECTED CONCRETE STRUCTURES
}

\author{
S.T. Yang ${ }^{1}$, K. F. $\mathrm{Li}^{2}$ and C.Q. $\mathrm{Li}^{3}$ \\ ${ }^{1}$ Department of Civil and Environmental Engineering, University of Strathclyde, Glasgow, \\ G1 1XJ, United Kingdom. \\ ${ }^{2}$ Department of Civil Engineering, Tsinghua University, Beijing, 100084, China. \\ ${ }^{3}$ School of Civil, Environmental and Chemical Engineering, RMIT University, GPO Box \\ 2476, Melbourne 3001, Australia.
}

\begin{abstract}
Corrosion-induced deterioration of reinforced concrete (RC) structures results in premature failure of the RC structures. In practice concrete crack width is one of the most important criteria for the assessment of the serviceability of RC structures. It is therefore desirable to predict the growth of the crack width over time so that better informed decisions can be made concerning the repairs due to concrete cracking. Literature review shows that little research has been undertaken on numerical prediction of concrete crack width. The intention of this study was to develop a numerical method to predict concrete crack width for corrosionaffected concrete structures. A cohesive crack model for concrete is implemented in the numerical formulation to simulate crack initiation and propagation in concrete. Choices for evaluating the parameters of cohesive elements are extensively discussed which is a key for developing a plausible model employing cohesive elements. The surface crack width is obtained as a function of service time. Accurate prediction of crack width can allow timely maintenance which prolongs the service life of the reinforced concrete structures.
\end{abstract}

Keywords: corrosion, crack width, reinforced concrete, numerical modelling.

* Corresponding author. Tel: +44 141548 3273. Email: shangtong.yang@strath.ac.uk. 
Reinforced concrete (RC) structures have been the most common type of structures used in the civil engineering construction since middle nineteenth century. RC structures have been widely used for building, bridges, retaining walls, tunnels, and indeed any physical infrastructure built on and under the ground. Since 1970s, it has become an accepted knowledge that the concrete cover has its limitation on protecting the reinforcing steel from corrosion. As a result, a series of research has been initiated on improving the understanding of the corrosion of steel in concrete [1], such as the Concrete in the Oceans research programme in the UK in the 1970s. Furthermore, it appears to be inevitable that RC structures will suffer from reinforcement corrosion in chloride $\left(\mathrm{Cl}^{-}\right)$and carbon dioxide $\left(\mathrm{CO}_{2}\right)$ laden environment. Practical experience and experimental observations [2-5] suggest that corrosion affected RC structures deteriorate faster in terms of serviceability (e.g., cracking or deflection) than safety (e.g., strength). Therefore, there is a well justified need for a thorough investigation of the cracking process and crack width of concrete, not least bearing in mind that crack width is one of the most important practical parameters for the design and assessment of RC structures.

To model cracking of concrete, some researchers have resorted to analytical approach, mainly due to the accuracy of the solution and the convenience of its practical application [6-8]. For example, Li and Yang [7] developed an analytical model for concrete crack width caused by reinforcement corrosion and applied load, by introducing a stiffness reduction factor to account for the post-cracking quasi-brittle behaviour of concrete. The stiffness reduction factor then modifies the differential equation for obtaining the cracked stress and strain components. Correlations between material corrosion and the structural effects can then be established, e.g., crack width [7], time to surface cracking [8], etc. However, the application 
of analytical modelling in crack propagation in concrete is limited to some special cases, e.g., particular boundary conditions, and the assumption that the crack is smeared and uniformly distributed in the damaged solid to satisfy the requirement on continuous displacement. Some studies have employed complex functions to formulate the stress development under arbitrary boundary conditions $[9,10]$; however, they have been limited to elastic problems only so far.

In light of the limitation of analytical modelling on crack propagation in concrete, numerical modelling has brought considerable advantages. Depending on the specific application and the scale of the problem, different numerical techniques may be used, e.g., finite element method (FEM) [11, 12], discrete element method (DEM) [13], boundary element method (BEM) $[14,15]$ and peridynamics $[16,17]$. Amongst these numerical methods, FEM has received the most research interest in solving corrosion-induced reinforced concrete cracking. Roesler et al. [11] developed a FE model with cohesive crack concept to predict the fracture performance of concrete beams. A number of geometrically similar beams were investigated and the global mechanical behaviour of the cracked beams was obtained. For corrosion induced concrete cracking, Guzman et al. [18] developed a concrete cover cracking model based on embedded cohesive crack finite element. Time to surface cracking was then able to be predicted. Sanchez et al. [19] proposed a mesoscopic model simulating the mechanical performance of reinforced beams affected by corrosion. Both cross-sectional and out of crosssection mechanisms, affected by corrosion, were coupled for determination of corrosion effects on the concrete structures. Moreover, Bossio et al. [20] considered the effects of corrosion of four reinforcing rebars on the behaviour of a single structural element. According to the research literature, however, there are very few models on numerical modelling of concrete crack width due to internal pressure such as corrosion induced expansion. Crack width is an important parameter regarding the durability of concrete structures while it is still not quite clear how those underlying factors, e.g., corrosion rate, material/mechanical 
properties of concrete, may quantitatively affect the development of crack width of the concrete. Therefore, it is well justified that a numerical method be developed to predict corrosion induced concrete crack width over service time.

This paper is based upon Yang et al. [21], but the current paper includes additional research in model formulation, i.e., cracking criteria, choice of parameters of cohesive elements and calculation of corrosion-induced displacement, and a parametric study, i.e., effects of numerical parameters on concrete crack width results. This paper attempts to develop a numerical method to predict the cracking and crack width for corrosion affected concrete structures. Cohesive crack model is used and cohesive elements are embedded for simulating the crack propagation. The choices of parameters of cohesive elements have been extensively discussed which is the key for establishing a plausible model with cohesive elements. After formulation of the model, an example is worked out to demonstrate the application of the method and verification by comparing with analytical results is provided. Parametric study is finally carried out to investigate the effects of some numerical parameters on the concrete crack width.

\section{CONSTITUTIVE MODEL}

The failure of structures is significantly influenced by the properties of the material used. In terms of tensile stress-elongation relationship, most of engineering materials can be classified into brittle, ductile and quasi-brittle [22]. Different materials used will result in different failure mechanisms of structures and hence different material models should be applied correspondingly. For example, Drucker-Prager Model and Von Mises Model are used for ductile materials. For brittle materials, Griffith model based on linear elastic fracture 
111 mechanics is usually applied. Cohesive Crack Model, one of few nonlinear fracture

112 mechanics models, is developed and widely used for quasi-brittle materials.

113

114 Concrete is considered as a quasi-brittle material, in which the tensile stress gradually

115 decreases after it reaches the tensile strength while the tensile strain/displacement continues to

116 increase. This behaviour of concrete is called strain softening. The concept of strain softening

117 evolves from plasticity where the post-peak decline of the tensile stress is considered as a

118 gradual decrease of the tensile strength, i.e., softening. Since the softening is related to all the

119 strain components, it is normally called strain softening. The reason of strain softening is that

120 there is an inelastic zone developed ahead of the crack tip which is also referred to as fracture

121 process zone (FPZ) as shown in Figure 1-a. When a crack propagates in concrete, the cracked

122 surfaces may be in contact and are tortuous in nature [23], due to various toughening

123 mechanisms such as aggregate bridging, void formation or microcrack shielding [22].

124 Therefore, the cracked surfaces may still be able to sustain the tensile stress which is

125 characterized by the softening degradation curve.

126

127 Cohesive Crack Model (CCM), originally developed by Hillerborg, et. al [24], is generally 128 accepted as a realistic simplification for FPZ [25]. CCM assumes that FPZ is long and narrow 129 and is characterized by a stress-displacement curve as typically shown in Figure 1-b. In 130 Figure 1-a, the shadowed zone from point A to B is FPZ and the area beyond Point B is the

131 true crack where the cracked surfaces are completely separated. The CCM is normally 132 incorporated into finite element analysis as an interface when the crack path is known in 133 advance. 
135 Since the FPZ is represented by the cohesive interface and the thickness of the cohesive

136 interface should be very small or zero, a traction-separation law is introduced to describe its

137 stress-displacement relationship as follows:

$$
\sigma=f_{T-S}(\delta)
$$

139 where $f_{T-S}$ is a nonlinear function, on which a number of researchers have been working to

140 define it. It has been found that with zero thickness, the traction-separation law for the 141 interface provides best estimation for concrete cracking because there is actually no real

142 interface in it. Since $\delta$ is related to cracking opening displacement $w, f_{T-S}(\delta)$ can also be

143 expressed in terms of $w$. As shown in Figure 1-b, there are four parameters to define $f_{T-S}(\delta)$ :

144 the elastic stiffness (also called penalty stiffness) $K_{p}$, the tensile strength $f_{t}^{\prime}$, the fracture 145 energy $G_{f}$ and the shape of the softening curve.

147 Since the crack opening $w$ can be determined via unloading process, the stress-displacement 148 relationship can also be expressed as stress-crack opening relationship. Thus the tractionseparation relation for exponential softening curve can be expressed as follows:

$$
\sigma=f(w)=f_{t}^{\prime} \exp \left(-\frac{f_{t}^{\prime}}{G_{f}} w\right)
$$

151 Once $f_{t}^{\prime}$ and $G_{f}$ are known, the constitutive relationship for the cohesive interface can be 152 determined.

154 As the cracking is assumed to occur at the interface, concrete outside the cracking zone, 155 known as bulk concrete, can be dealt with by linear elastic mechanics. Once a crack occurs, 156 the bulk concrete undergoes unloading. The stress-strain relationship for the bulk concrete is 157 linear as shown below: 


$$
\sigma^{\prime}=E \varepsilon^{\prime}
$$

161 where $\sigma^{\prime}$ represents tensile/compressive stress and $\varepsilon^{\prime}$ represents the corresponding strain.

162

163 Penalty stiffness $K_{p}$ : since $f(w)$ defines only the strain softening after the peak stress $f_{t}^{\prime}$, the 164 elasticity of the concrete prior to the peak stress needs to be described separately. The initial 165 response of the cohesive interface is assumed to be linear and represented by a constant 166 penalty stiffness $\left(K_{p}\right)$ as shown in Figure 1-b. The concept of penalty stiffness comes from 167 the elastic stiffness which is obtained by dividing the elastic modulus of the concrete by its 168 thickness. Since cohesive interface is normally very thin or even of zero thickness, the elastic 169 stiffness of the cohesive interface approaches infinitesimally large. This makes sense as the 170 interface should be stiff enough prior to initiation of crack to hold the two surfaces of the bulk 171 concrete together, leading to the same performance as that of no interface existing. This also 172 meets the condition of CCM which assumes that the energy required to create the new 173 surfaces is vanishingly small compared to that required to separate them [26]. The reason for 174 this condition is that when the elastic stiffness is large, the displacement at tensile strength is 175 small and thus the energy to create the new surfaces is small. However, the elastic stiffness 176 cannot be too large as it will cause convergence problems due to ill-conditioning of the 177 numerical solver of the FE programmes [27]. Therefore, the cohesive stiffness becomes a 178 "penalty" parameter $\left(K_{p}\right)$, which controls how easily the cohesive interface deforms 179 elastically. As such this stiffness is large enough to provide the same or close response of 180 intact concrete prior to cracking, but not so large as to cause numerical problems. 
182 Tensile strength $f_{t}^{\prime}$ : The tensile strength $f_{t}^{\prime}$ of concrete material is used as an important index 183 to determine if a cohesive crack is initiated. For Mode I fracture, once the tensile stress at any 184 point of a structure reaches its tensile strength, a crack is initiated and the material of that 185 point starts to degrade. As is known, the tensile strength of concrete can be obtained mainly 186 by three types of tests, which are splitting test, flexural test and direct tensile test. The 187 strengths measured from these tests vary considerably and $f_{t}^{\prime}$ must be determined via direct 188 tensile test. This is because, in the splitting and flexural tests, the distributed stresses are not 189 pure tension but involving compression. The strength determined from such tests, therefore, is 190 not truly tensile property of concrete.

192 Fracture energy $G_{f}$ : The fracture energy $G_{f}$ is the energy absorbed per unit area of crack 193 with the unit of $\mathrm{N} / \mathrm{mm}$ or $\mathrm{N} / \mathrm{m}$. It can be regarded as the external energy supply required to 194 create and fully break a unit surface area of cohesive crack. Therefore, $G_{f}$ can be calculated 195 as the area under the softening curve shown in Figure 1-b and expressed as follows 196

$$
G_{f}=\int_{0}^{\delta_{m}} f_{T-S}(\delta) d \delta
$$

198 Since the entire stress-displacement curve $f_{T-S}(\delta)$ is regarded as a material property, $G_{f}$ is also 199 a material parameter which is independent of structural geometry and size. $G_{f}$ is used as an energy balance which controls stable crack propagation, that is, a crack will propagate when 201 the strain energy release rate is equal to $G_{f}$.

203 Shape of softening curve: The cohesive crack initiation is followed by strain softening, which 204 can be represented by a range of forms, e.g., linear, bilinear and non-linear softening. Without 
knowing the shape of the softening curve, it is difficult to determine the entire stress-

206 displacement curve. Although some researchers have suggested that the exact shape of the 207 softening curve is less important than the values of fracture energy for certain cases [28], the 208 shape of the softening curve is important in predicting the structural response and the local 209 fracture behaviour, i.e. the crack width is particularly sensitive to the shape of the softening 210 curve [22].

\section{FE Simulation}

2134 nodes cohesive interface element which has two stress components - normal stress in 214 direction 1 and shear stress in direction 2 is used in the simulation. There are no other stresses 215 because the thickness in direction 1 is infinitesimally small.

This cohesive interface element will have linear elastic behaviour prior to the peak stress, i.e., tensile strength, followed by the initiation and evolution of damage, i.e., cracking. The elastic constitutive relationship between the nominal stresses and nominal strains is described as 221 follows:

$$
\sigma=\left\{\begin{array}{l}
\sigma_{1} \\
\sigma_{2}
\end{array}\right\}=\left[\begin{array}{ll}
E & 0 \\
0 & G
\end{array}\right]\left\{\begin{array}{l}
\varepsilon_{1} \\
\varepsilon_{2}
\end{array}\right\}
$$

where $\sigma_{1}$ and $\sigma_{2}$ are the normal stress in direction 1 and shear stress in direction 2 respectively, $G$ is the shear modulus in plane state (in 2D), and $\varepsilon_{1}$ and $\varepsilon_{2}$ are the corresponding strains of $\sigma_{1}$ and $\sigma_{2}$.

For concrete with embedded reinforcing steel bar, it is widely accepted to be modelled as a 
231 placement of cohesive interface. It is assumed that only one crack will initiate and propagate

232 from the inner boundary of the cylinder to the outer boundary. However, this crack represents

233 the total cracks in a way that the total crack width can be divided by the number of the cracks,

234 as widely employed in smeared crack model. For FEA, two elements are employed in this

235 study: 4 nodes cohesive interface element as discussed earlier for the cohesive interface, and 4

236 nodes bilinear plane strain quadrilateral element for the bulk concrete. Reduced integration is

237 used for the plane strain element because the accuracy of the bulk concrete is not an issue. As

238 a result, the damage evolution of the cohesive element is combined with the elastic

239 deformation of the bulk concrete in the global response.

240

241

242

243

Additionally, very fine mesh is used in the cohesive interface and its surrounding bulk concrete. The thickness of the cohesive interface is $0.2 \mathrm{~mm}$ and the inner radius and outer radius are $6 \mathrm{~mm}$ and $37 \mathrm{~mm}$ respectively. Since the cohesive interface should only accommodate a single layer of cohesive elements due to traction-separation law, the element size of the cohesive element is chosen as $0.2 \mathrm{~mm}$. The region around the cohesive interface will have stress concentration during the cracking process of the cohesive elements which should have the same element size as the cohesive element. The other area of the bulk concrete is in pure linear elasticity and has no concentration of stress; therefore, much coarser mesh can be applied. It has been tried on this selected mesh size to ensure that the convergence is not the problem due to the mesh size.

The cylinder is subjected to a uniformly distributed pressure at the inner boundary, i.e., the corrosion induced pressure and applied load induced pressure. For brittle and ductile materials, pressure/force can be directly applied to the boundary. However, for strain softening materials, only displacement can be used as boundary condition. This is because, the far field force/stress, does not monotonically increase; instead, it will drop after initial 

applied as boundary condition for strain-softening materials. In this model, the expansion cannot be just uniformly distributed due to the introduction of the cohesive interface. The reason is that if the radial displacement is applied uniformly in a solar coordinate system, 262 there will be a component in the normal direction (direction 1 in Fig. 4-3) of the $1^{\text {st }}$ cohesive element at the inner boundary because of its finite geometric thickness, which is illustrated in

264 Figure 4. The component can only be waived if the cohesive elements are geometrically 265 modelled as zero thickness, which will lead to the expansion in Figure 4 in horizontal 266 direction. Such a displacement component results in dramatically large stress since the 267 stiffness of the cohesive elements are much larger than the surrounding bulk concrete.

Due to the fact that the displacement (normal component) cannot be directly applied to the 1st 270 cohesive element, the displacement is applied in two coordinate systems in this study. The 271 displacement applied to the cohesive element is defined in direction of x-axis in rectangular 272 coordinate system, and the displacement applied to the other part of the inner boundary is defined in radial direction in cylindrical coordinate system. With this arrangement, the

274 geometric thickness of the cohesive element needs to be very small. This arrangement 275 eliminates the normal component of the displacement on the $1^{\text {st }}$ cohesive element and 276 approximately reserves the shear component of the displacement. Since the thickness of the 277 cohesive element is extremely small, the shear component of the uniformly distributed 278 displacement can be considered the same as the distributed displacement itself. Under this 279 arrangement, the traction of the cohesive element comes from the deformation of the whole 280 cylinder and there is no artificial displacement added to the normal direction of the cohesive 281 element. 
The inner displacement boundary condition of the concrete is caused by reinforcement 284 corrosion which can be calculated by analytical means. According to Li and Yang [7] 285 formulated the corrosion-induced reinforcement expansion volume and the displacement at 286 the inner boundary of the concrete. Details about the analytical formulation can be referred to $\mathrm{Li}$ and Yang [7] while the corrosion-induced displacement of expansion $d_{c}(t)$ is listed as 288 follows:

289

$$
d_{c}(t)=\frac{W_{\text {rust }}(t)}{\pi D}\left(\frac{1}{\rho_{\text {rust }}}-\frac{\alpha_{\text {rust }}}{\rho_{\text {st }}}\right)-d_{0}
$$

291

292

293

294

where $D$ is diameter of the reinforcing rebar, $d_{0}$ is the thickness of the interfacial porous band between concrete and reinforcement, $\alpha_{\text {rust }}$ is the molecular weight of steel divided by the molecular weight of corrosion products. It varies from 0.523 to 0.622 according to different types of corrosion products [30]. $\rho_{r u s t}$ and $\rho_{s t}$ are the densities of corrosion products and the original steel, respectively. $W_{\text {rust }}(t)$ is related to the corrosion rate of the steel rebar and can be expressed as follows [7]:

$$
W_{\text {rust }}(t)=\sqrt{2 \int_{0}^{t} 0.105\left(1 / \alpha_{\text {rust }}\right) \pi D i_{\text {corr }}(t) d t}
$$

where $i_{\text {corr }}$ is the corrosion current density in $\mu \mathrm{A} / \mathrm{cm}^{2}$, which is widely used as a measure of corrosion rate.

By using Equations (6) and (7), the time-dependent displacement of the inner boundary of the concrete cylinder can be obtained for FE analysis, as illustrated in Figure 5. 
Crack initiation marks the beginning of degradation or damage of concrete at a point. Crack is assumed to initiate when the maximum nominal tensile stress reaches the tensile strength of the concrete for the Mode I fracture - opening mode, expressed as follows

$$
\left\langle\sigma_{1}\right\rangle=f_{t}^{\prime}
$$

314 where $\left\langle\sigma_{1}\right\rangle=\left\{\begin{array}{ccc}\sigma_{1} & \text { for } & \sigma_{1}>0 \\ 0 & \text { for } & \sigma_{1}<0\end{array}\right.$

The operation $\left\langle\sigma_{1}\right\rangle$ is to ensure that a crack will not initiate under compression.

After cracking is initiated, the cohesive element is damaged and the normal stress of this element softens in a manner as defined (e.g., Figure 1b). The failure of the element is governed by the softening curve. To calculate the residual stress after its peak/cracking stress, a damage parameter $D$ is introduced into the stress calculation as follows:

where $\sigma_{u}$ is the undamaged stress as shown in Figure 6.

To prevent mesh sensitivity in FE analysis, the damage evolution has to be based on displacement or energy rather than strain. This means the crack opening is not dependent on the strain of the element but the opening distance of the element. Therefore, as the distance

331 between the nodes is used as a crack measure rather than a change in strain (which depends on

332 the element length) the mesh dependency is significantly reduced.

334 To calculate the residual stress after its peak/cracking stress, a damage parameter D is defined 335 as follows 
where $G_{r}$ is the energy release rate after peak stress, $G_{e}$ is the elastic energy release rate prior to peak stress. These energy parameters are illustrated in Figure 7.

342 Convergence is usually a problem in the execution of FE programmes for materials exhibiting 343 softening behaviour for implicit scheme as in most FE programmes. Also, when a material is 344 damaged, e.g., concrete is cracked, sudden dissipation of energy will make the computation more dynamical while the quasi-static analysis is expected. An artificial viscosity is therefore used to overcome the convergence difficulties by making the stiffness matrix of the material positive. This viscosity regularizes the traction-separation law by modifying the stiffness reduction variable $D$ as follows

$$
\dot{D_{v}}=\frac{D-D_{v}}{\mu}
$$

where $\mu$ is the viscosity parameter which can be specified in the property of cohesive element and $D_{v}$ is the viscous stiffness degradation variable. Once $\mu$ and $D$ are known, $D_{v}$ can be determined. A small viscosity value $\mu$ helps improve the rate of convergence without compromising results.

\section{Worked Example}

357 As a demonstration of the application of the developed numerical method and techniques in 358 FEA, the example used in $\mathrm{Li}[3]$ is taken for numerical solutions. The loading is applied to the 359 concrete in the form of displacement rather than pressure, due to the strain softening 
behaviour as explained previously. Figure 5 shows the displacement applied to the concrete as

361 a function of service time which can be calculated analytically using classic mechanics. In this

362 example, the stress-displacement relationship is taken from the direct tensile test, as shown in 363 Figure 7.

365 The values of the basic variables used in the numerical solution are listed in Table 1. To 366 calculate the effective modulus of elasticity, the creep coefficient is taken as 2.0. Since the 367 cohesive element size is of $0.0002 \mathrm{~m}$ and the theoretical thickness of the cohesive element is 3681 , the elastic stiffness of the cohesive interface is $35250 \mathrm{GPa}\left(5000 E_{e f}\right)$. However, due to the 369 value is too large, the penalty stiffness is taken as $14100 \mathrm{GPa}\left(2000 E_{e f}\right)$. The time-dependant 370 internal displacement, i.e., Figure 5, is applied to the concrete cylinder as the boundary 371 displacement condition. The constitutive stress-displacement relation is obtained from the 372 direct tensile test on concrete. The stress-inelastic effective displacement curve can be plotted 373 in Figure 8.

375 The crack finally approaches the outer boundary of the cylinder (surface). Since the 376 theoretical thickness of the cohesive element is set to be 1.0, the strain of the cohesive 377 element is equal to its displacement. Upon removing the elastic displacement from the total 378 displacement of the last cohesive element at the outer boundary of the cylinder, the surface 379 crack width can be expressed in a function of time, shown in Figure 9.

381 In Figure 9, it can be seen that the surface crack width increases with time. The abrupt 382 increase in the crack width corresponds to rapid decrease of tensile stress, or sudden energy 383 release, in the element as shown in Figure 8. After about 4 years, the increase of the crack 384 width is steady and seems to approach certain value after about 7 years. This might be due to 
a combined effect of the steady decrease of the tensile stress (long tail of the stress-

displacement curve in Figure 8) and the nonlinear development of displacement applied at the

inner boundary (i.e., Figure 5). At 10 years, the crack width reaches about $0.23 \mathrm{~mm}$.

To verify the proposed numerical method, the results are compared with those from the recently developed analytical model [7]. By using the same inputs, which are mainly from Li

391 [31] and Liu and Weyers [30], the resulted crack width from both methods can be compared 392 as a function of service time, as shown in Figure 10. It can be seen that the numerical results 393 are in good agreement with the analytical results.

As discussed, the results of materials exhibiting softening behaviour and degradation of stiffness will normally have severe convergence problems. A common numerical technique to 397 solve the convergence difficulty is to employ a small viscosity value to regularize the 398 constitutive equations, as presented in Equation 9. Figure 11 shows the effect of the viscous regularization on the predicted concrete crack width with three viscosity values used. Visco5e-4, Visco1e-3 and Visco5e-3 represent viscosity values of 5e-4, 1e-3 and 5e-3 respectively. The analytical result [7] is also plotted in Figure 11 for comparison. Smaller 402 viscosity values, i.e. 1e-4, have been used but no converged results have been obtained. It can 403 be seen from Figure 11 that the viscosity value of $5 \mathrm{e}-4$ matches best with the analytical 404 results. Higher viscosity values provide better convergence, i.e., easier to converge and less 405 increments required, but also affect the results more than the lower values of viscosity. 406 Therefore, the viscosity coefficient should be kept as small as it can make the analysis be 407 converged. In this example, the appropriate value of viscosity coefficient is considered as 5e4084. 
411 Penalty stiffness is the cohesive stiffness as shown in Figure 1b which controls how easily the 412 cohesive interface deforms elastically. To investigate its effect on the results of concrete crack 413 width, three values of penalty stiffness are employed and the results are shown in Figure 12.

414 Penalty1, Penalty2 and Penalty3 represent the values of penalty stiffness of $14100 \mathrm{GPa}, 7050$

$415 \mathrm{GPa}$ and $3525 \mathrm{GPa}$ respectively. $14100 \mathrm{GPa}$ was used in the worked example. It can be seen 416 that smaller penalty stiffness makes the surface cracking time earlier. There might be 417 confusion herein that the penalty stiffness controls the elasticity of the cohesive elements but 418 it does affect the concrete crack width which is mainly controlled by the inelastic behaviour 419 of the cohesive elements. This can be explained by using Figure 6 that the calculation of the 420 residual tensile stress is dependent on the undamaged stress $\sigma_{u}$ which is determined by the 421 penalty stiffness. Therefore the energy required to break a unit cohesive surface (fracture 422 energy) is influenced by the penalty stiffness. It thus explains why the early stage of cracking, 423 i.e., surface cracking initiation, is sensitive to the change of penalty stiffness. However, the 424 long-term development of crack width seems not affected by the penalty stiffness. The reason 425 for that could be the long-term development of crack width is considerably influenced by the 426 tail of the stress-displacement curve as shown in Figure 6. The tail of the curve is, however, 427 negligibly affected by the penalty stiffness.

428

429

430

\section{Conclusions}

A numerical method to predict the crack width induced by reinforcement corrosion has been developed based on fracture mechanics and using finite element method. The concept of cohesive process zone has been employed to model the cracking behaviour of concrete whose constitutive relationship is characterised by a traction-separation law. A worked example has been presented to first demonstrate the application of the derived method and then compare with the results from an analytical method as a means of verification. It has been found that 
the numerical results are in good agreement with the analytical results, with an average difference of $4 \%$ within 10 years. It can be concluded that the numerical method presented in the paper can predict the concrete crack width induced by reinforcement corrosion with reasonable accuracy.

\section{Acknowledgement}

The authors wish to acknowledge the support of the European Commission via the Marie Curie IRSES project GREAT - Geotechnical and geological Responses to climate change: Exchanging Approaches and Technologies on a world-wide scale (FP7-PEOPLE-2013IRSES- 612665). Financial support from Scottish Funding Council GRPe for early career researcher exchanges is also gratefully acknowledged.

\section{References}

[1] N. J. M. Wilkins, and P. F. Lawrence, Concrete in the Oceans: Fundamental Mechanics of Corrosion of Steel Reinforcements in Concrete Immersed in Sea Water, Slough, UK, 1980.

[2] C. Andrade, F. J. Molina, and C. Alonso, "Cover Cracking as A Function of Rebar Corrosion: Part 1 - Experiment Test," Materials and Structures, vol. 26, pp. 453-454, 1993.

[3] C. Q. Li, "Life-Cycle Modelling of Corrosion-Affected Concrete Structures: Propagation," ASCE Journal of Structural Engineering, vol. 129, no. 6, pp. 753-761, 2003.

[4] C. Q. Li, "Time Dependent Reliability Analysis of the Serviceability of Corrosion Affected Concrete Structures," International Journal of Materials and Structural Reliability, vol. 3, no. 2, pp. 105-116, 2005.

[5] N. Otsuki, S. Miyazato, N. B. Diola et al., "Influences of Bending Crack and WaterCement Ratio on Chloride-Induced Corrosion of Main Reinforcing Bars and Stirrups," ACI Materials Journal, vol. 97, no. 4, pp. 454-465, 2000.

[6] S. J. Pantazopoulou, and K. D. Papoulia, "Modeling cover cracking due to reinforcement corrosion in RC structures," Journal of Engineering Mechanics, ASCE, vol. 127, no. 4, pp. 342-351, 2001.

[7] C. Q. Li, and S. T. Yang, "Prediction of Concrete Crack Width under Combined Reinforcement Corrosion and Applied Load," Journal of Engineering Mechanics, ASCE, vol. 137, no. 11, pp. 722-731, Nov, 2011. 
[8] K. Bhargava, A. K. Ghosh, Y. Mori et al., "Model for cover cracking due to rebar corrosion in RC structures," Engineering Structures, vol. 28, no. 8, pp. 1093-1109, 7//, 2006.

[9] S. T. Yang, K. F. Li, and C. Q. Li, "Non-uniform corrosion-induced reinforced concrete cracking: an anlytical approach." Magazine of Concrete Research, in press.

[10] X. Ning, R. Qingwen, P. Joe et al., "Nonuniform Corrosion-Induced Stresses in SteelReinforced Concrete," 2012/04/01, 2012.

[11] J. Roesler, G. H. Paulino, K. Park et al., "Concrete Fracture Prediction Using Bilinear Softening," Cement and Concrete Composite, vol. 29, pp. 300-312, 2007.

[12] J. H. Hanson, and A. R. Ingraffea, "Using Numerical Simulations to Compare the Fracture Toughness Values for Concrete from the Size-Effect, Two-Parameter and Fictitious Crack Models," Engineering Fracture Mechanics, vol. 70, pp. 1015-1027, 2003.

[13] B. Beckmann, K. Schicktanz, D. Reischl et al., "DEM simulation of concrete fracture and crack evolution," Structural Concrete, vol. 13, no. 4, pp. 213-220, 2012.

[14] M. H. Aliabadi, and A. L. Saleh, "Fracture mechanics analysis of cracking in plain and reinforced concrete using the boundary element method," Engineering Fracture Mechanics, vol. 69, no. 2, pp. 267-280, 1//, 2002.

[15] A. H. Chahrour, and M. Ohtsu, "BEM Analysis of Crack Propagation in Concrete Based on Fracture Mechanics," Boundary Element Methods: Fundamentals and Applications, S. Kobayashi and N. Nishimura, eds., pp. 59-66, Berlin, Heidelberg: Springer Berlin Heidelberg, 1992.

[16] D. Huang, G. Lu, and Y. Liu, "Nonlocal Peridynamic Modeling and Simulation on Crack Propagation in Concrete Structures," Mathematical Problems in Engineering, vol. 2015, pp. 11, 2015.

[17] W. Gerstle, N. Sau, and S. Silling, "Peridynamic modeling of concrete structures," Nuclear Engineering and Design, vol. 237, no. 12-13, pp. 1250-1258, 7//, 2007.

[18] S. Guzmán, J. C. Gálvez, and J. M. Sancho, "Modelling of corrosion-induced cover cracking in reinforced concrete by an embedded cohesive crack finite element," Engineering Fracture Mechanics, vol. 93, pp. 92-107, 10//, 2012.

[19] P. J. Sánchez, A. E. Huespe, J. Oliver et al., "Mesoscopic model to simulate the mechanical behavior of reinforced concrete members affected by corrosion," International Journal of Solids and Structures, vol. 47, no. 5, pp. 559-570, 3/1/, 2010.

[20] A. Bossio, T. Monetta, F. Bellucci et al., "Modeling of concrete cracking due to corrosion process of reinforcement bars," Cement and Concrete Research, vol. 71, pp. 78-92, 5//, 2015.

[21] S. T. Yang, K. F. Li, and C. Q. Li, "Numerical model for concrete crack width caused by reinforcement corrosion and applied load," in 15th International Conference on Civil, Structural and Environmental Engineering Computing, Prague, Czech Republic, 2015.

[22] S. P. Shah, S. E. Swartz, and C. Ouyang, Fracture Mechanics of Concrete: Applications of Fracture Mechanics to Concrete, Rock, and Other Quasi-brittle Materials, p.^pp. 552, New York: John Wiley \& Sons, Inc., 1995.

[23] S. Mindess, and S. Diamond, "The Cracking and Fracture of Mortar," Materials and Structures, vol. 15, no. 86, pp. 107-113, 1982.

[24] A. Hillerborg, M. Modeer, and P. E. Petersson, "Analysis of crack formation and crack growth in concrete by means of fracture mechanics and finite elements," Cement and Concrete Research, vol. 6, no. 6, pp. 773-781, 1976.

[25] M. Elices, C. Rocco, and C. Rosello, "Cohesive Crack Modeling of A Simple Concrete: Experimental and Numerical Results," Engineering Fracture Mechanics, vol. 76, no. 10, pp. 1398-1410, 2009. 
[26] Z. P. Bažant, and J. Planas, Fracture and Size Effect in Concrete and Other Quasibrittle Materials, Boca Raton, Florida: CRC Press, 1998.

[27] A. C. 446.3R, Finite Element Analysis of Fracture in Concrete Structures, Farmington Hills, Mich, 1997.

[28] M. Elices, G. V. Guinea, J. Gomez et al., "The Cohesive Zone Model: Advantages, Limitations and Challenge," Engineering Fracture Mechanics, vol. 69, pp. 137-163, 2002.

[29] R. Tepfers, "Cracking of concrete cover along anchored deformed reinforcing bars," Magazine of Concrete Research, vol. 31, no. 106, pp. 3-12, 1979.

[30] Y. Liu, and R. E. Weyers, "Modelling the time-to-corrosion cracking in chloride contaminated reinforced concrete structures," ACI Materials Journal, vol. 95, no. 6, pp. 675-681, 1998.

[31] C. Q. Li, "Life cycle modelling of corrosion affected concrete structures propagation," Journal of Structural Engineering, ASCE, vol. 129, no. 6, pp. 753-761, 2003. 
541

542 1. Values of basic variables used in the example

543

544

545 
Table 1 Values of basic variables used in the example

548

\begin{tabular}{cccc}
\hline Description & Symbol & Values & Sources \\
\hline Inner radius & $a$ & $6 \mathrm{~mm}$ & $\mathrm{Li}[3]$ \\
Outer radius & $b$ & $37 \mathrm{~mm}$ & $\mathrm{Li}[3]$ \\
Effective modulus of Elasticity & $E_{e f}$ & $7.05 \mathrm{GPa}$ & Experiment \\
Poisson's ratio & $v_{c}$ & 0.18 & $\mathrm{Li}[3]$ \\
Tensile strength & $f_{t}^{\prime}$ & $1.7 \mathrm{MPa}$ & Experiment \\
Fracture energy & $G_{f}$ & $65 \mathrm{~N} / \mathrm{m}$ & Experiment \\
\hline
\end{tabular}

549

550 


\section{LIST OF FIGURES}

553 1. Cohesive crack model for the FPZ

554 2. Local directions for the two-dimensional cohesive element

555 3. Geometry of the FE model and the mesh around the cohesive interface

556 4. Stresses of the $1^{\text {st }}$ cohesive element under uniform load distribution

557 5. Internal expansion (displacement) as function of service time

558 6. Determination of residual stress in terms of the damage parameter $D$

559 7. Illustration of various energy release rates

560 8. Constitutive relation inputs for CCM used in the example

561 9. Crack width as a function of time

562 10. Crack widths as a function of time by both methods

563 11. Effect of viscous regularization on the predicted concrete crack width

564 12. Effect of penalty stiffness on predicted concrete crack width 


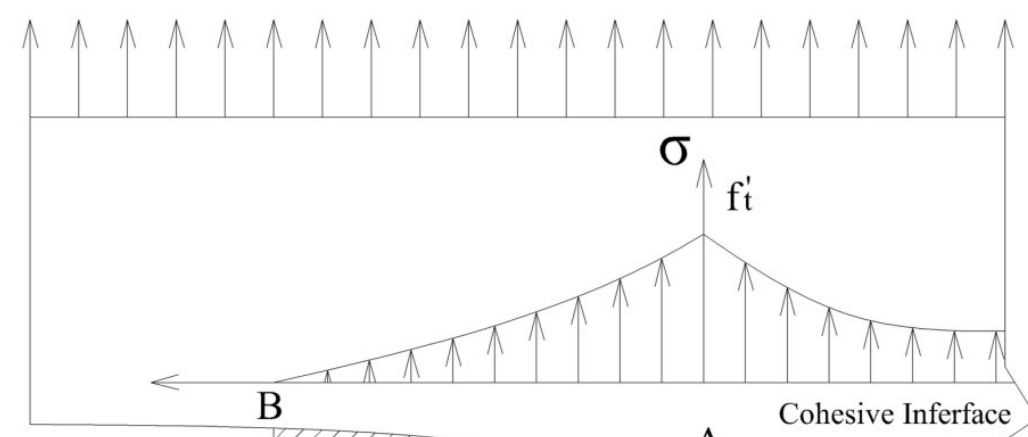

(a) Schematic of mechanism of FPZ

573

574

575

576

577

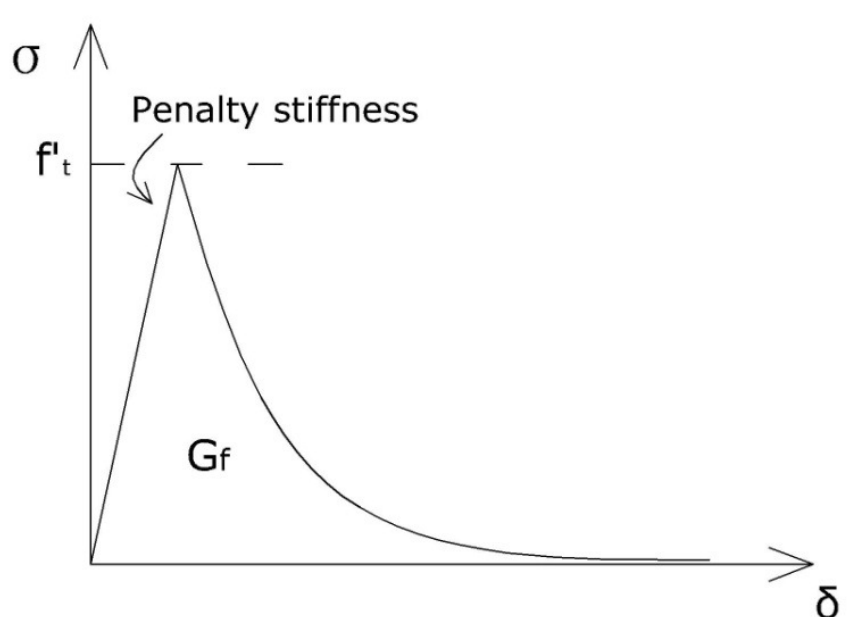

(b) Stress-displacement curve for cohesive material

Figure 1 Cohesive crack model for the FPZ 


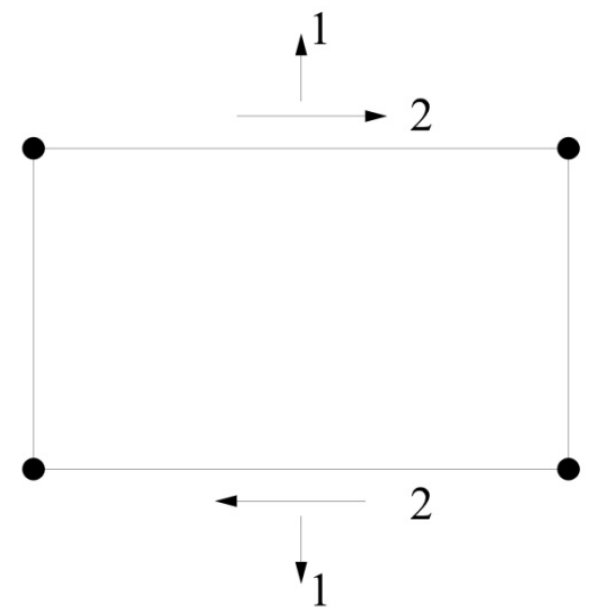

580

581

Figure 2 Local directions for the two-dimensional cohesive element 
582

583

584 585

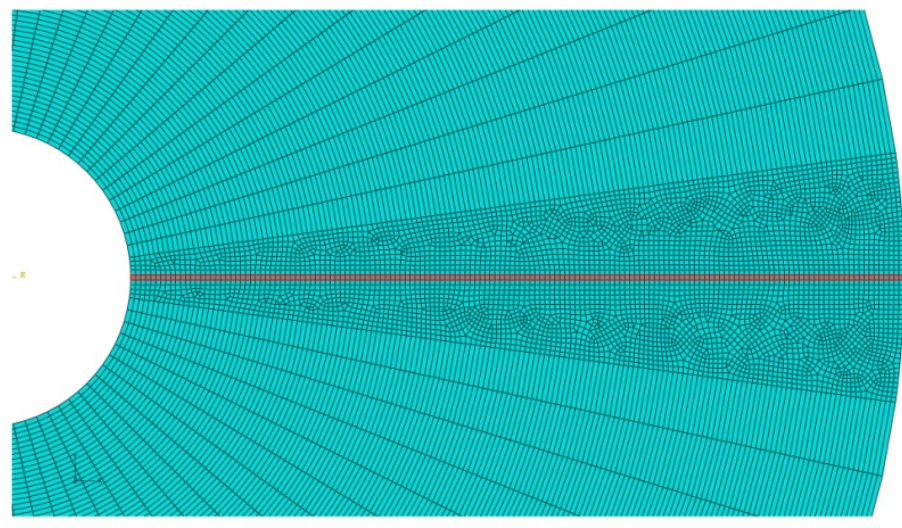

Figure 3 Geometry of the FE model and the mesh around the cohesive interface 


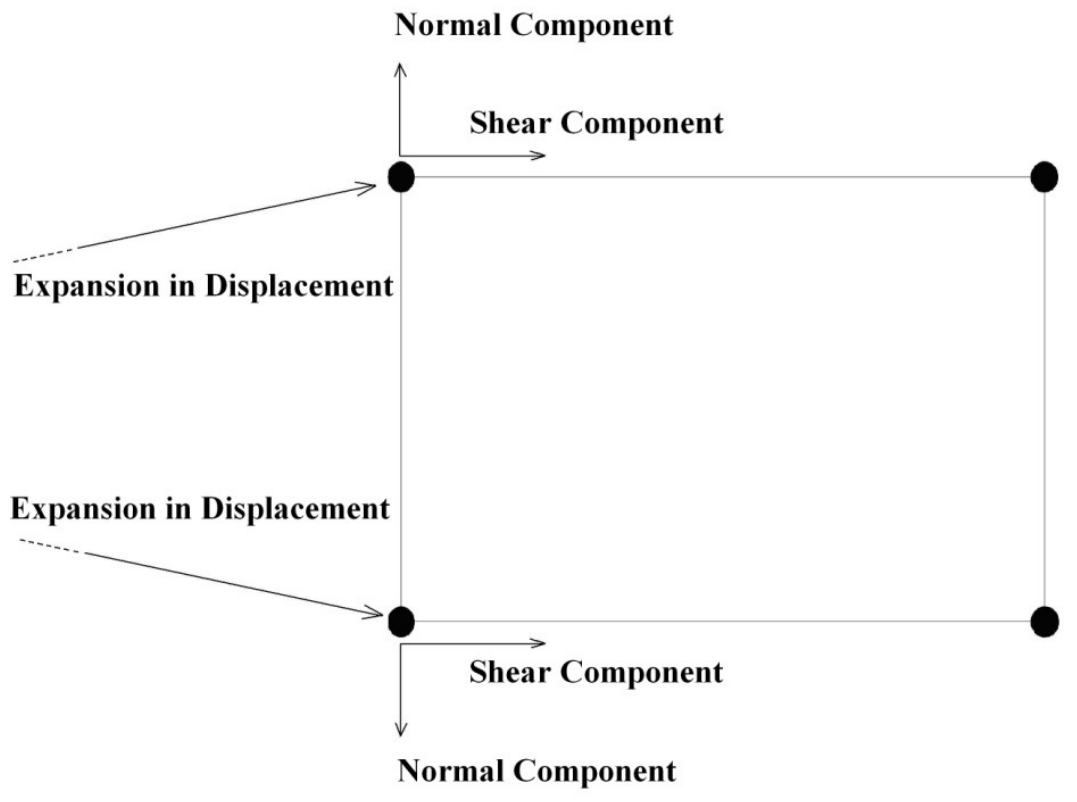

Figure 4 Stresses of the $1^{\text {st }}$ cohesive element under uniform load distribution 


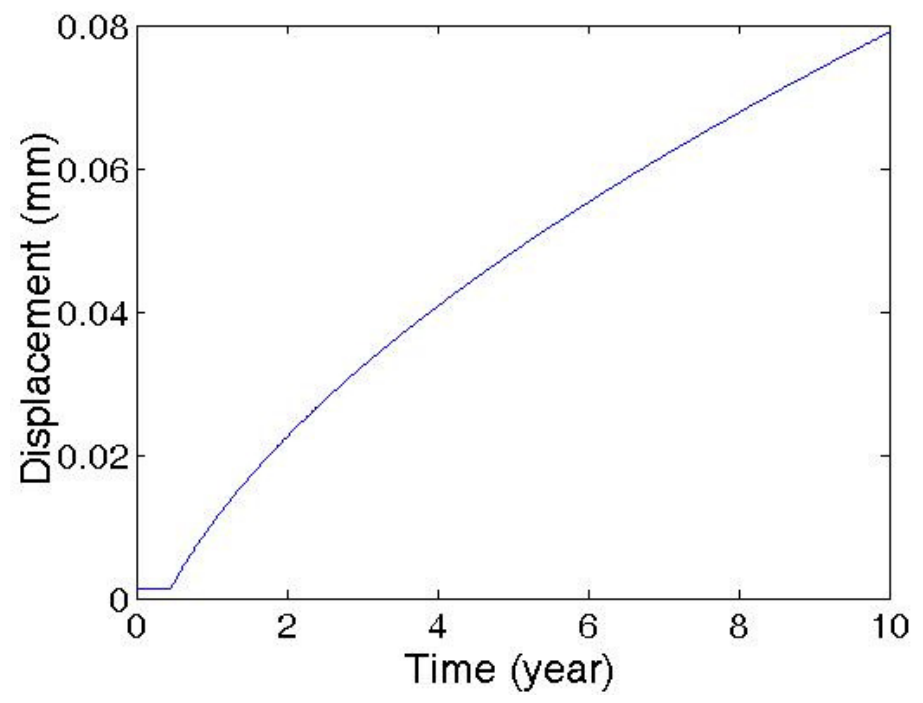

Figure 5 Internal expansion (displacement) as function of service time 594 


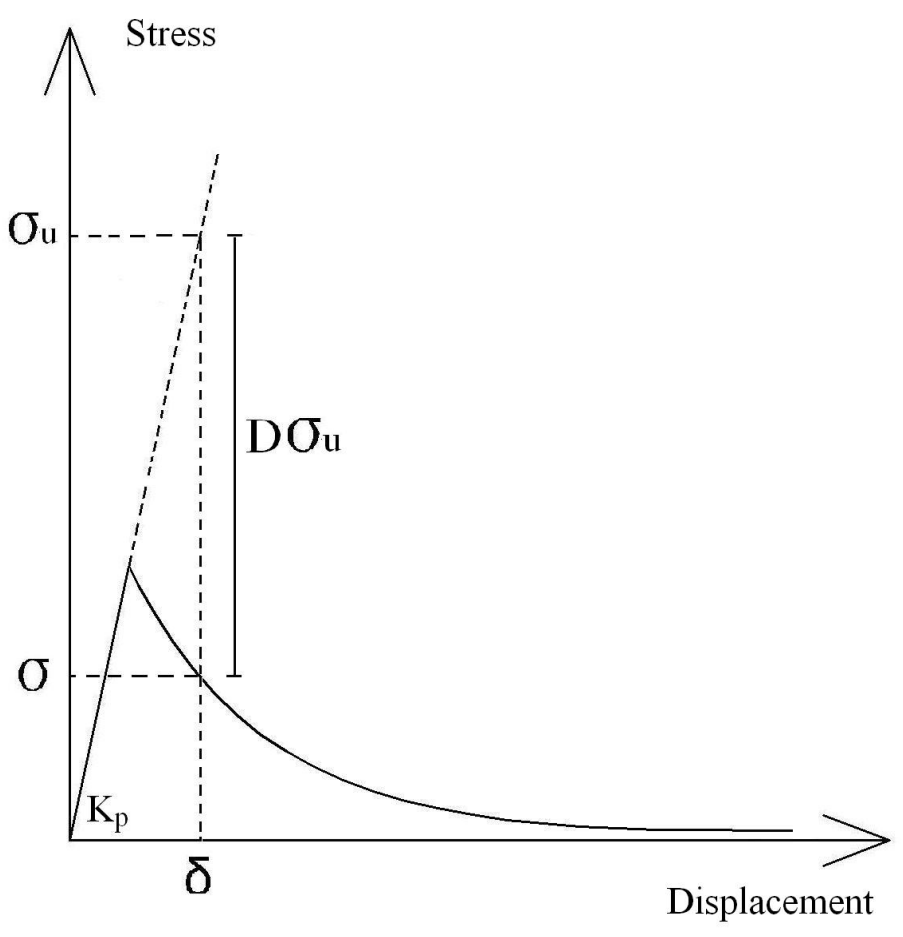

Figure 6 Determination of residual stress in terms of the damage parameter $D$ 
600

601

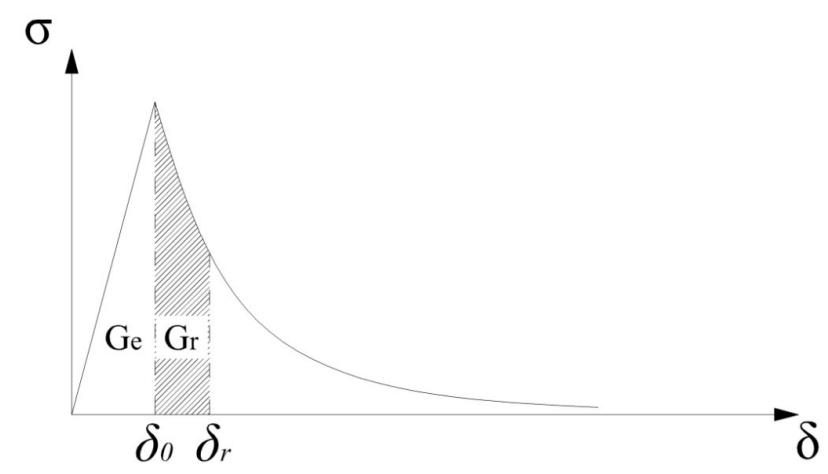

602

603

Figure 7 Illustration of various energy release rates

604 
605

606

607

608

609

610

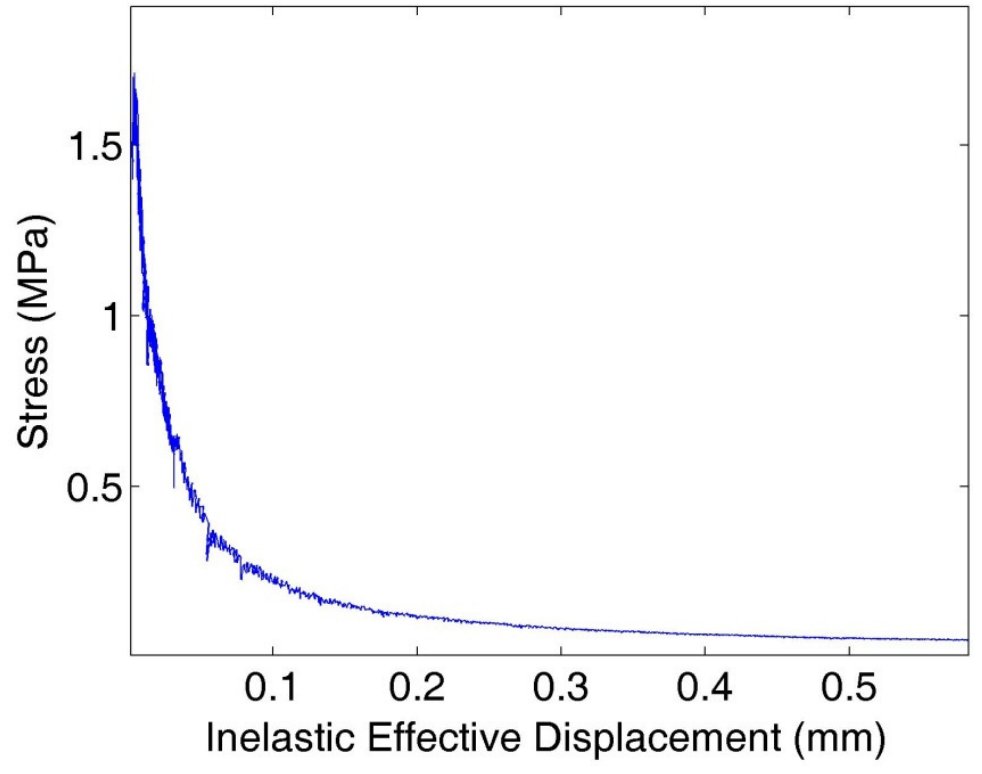

Figure 8 Constitutive relation inputs for CCM used in the example 
611

612

613

614

615

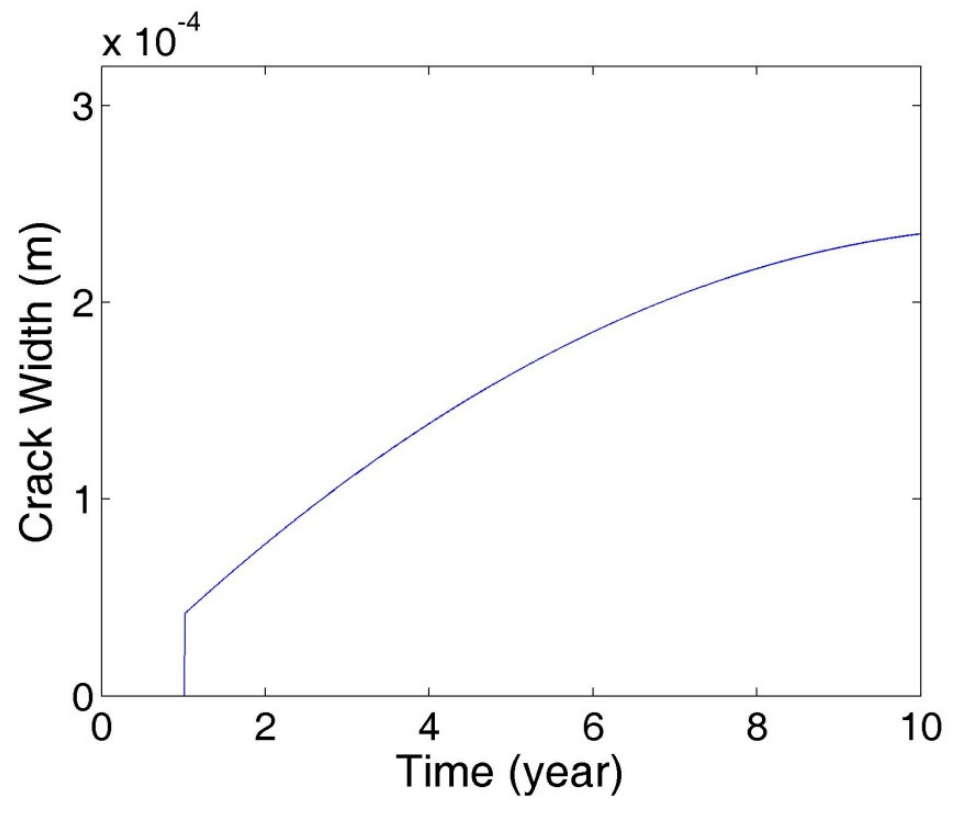

Figure 9 Crack width as a function of time 


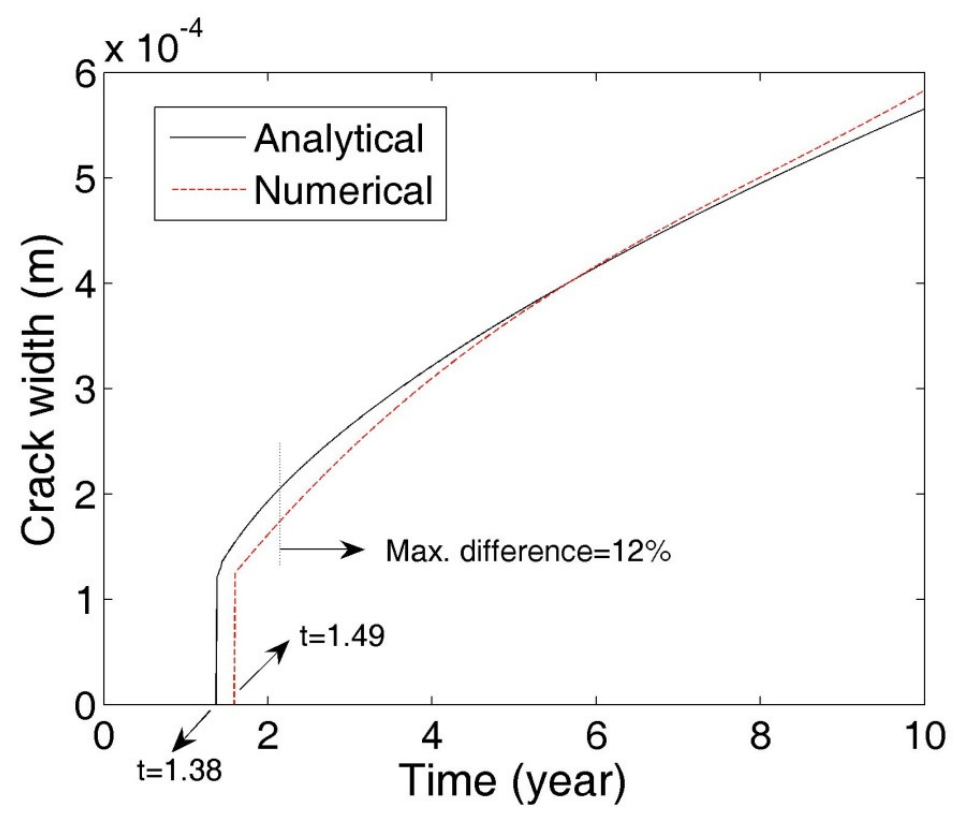

617

618

Figure 10 Crack widths as a function of time by both methods 


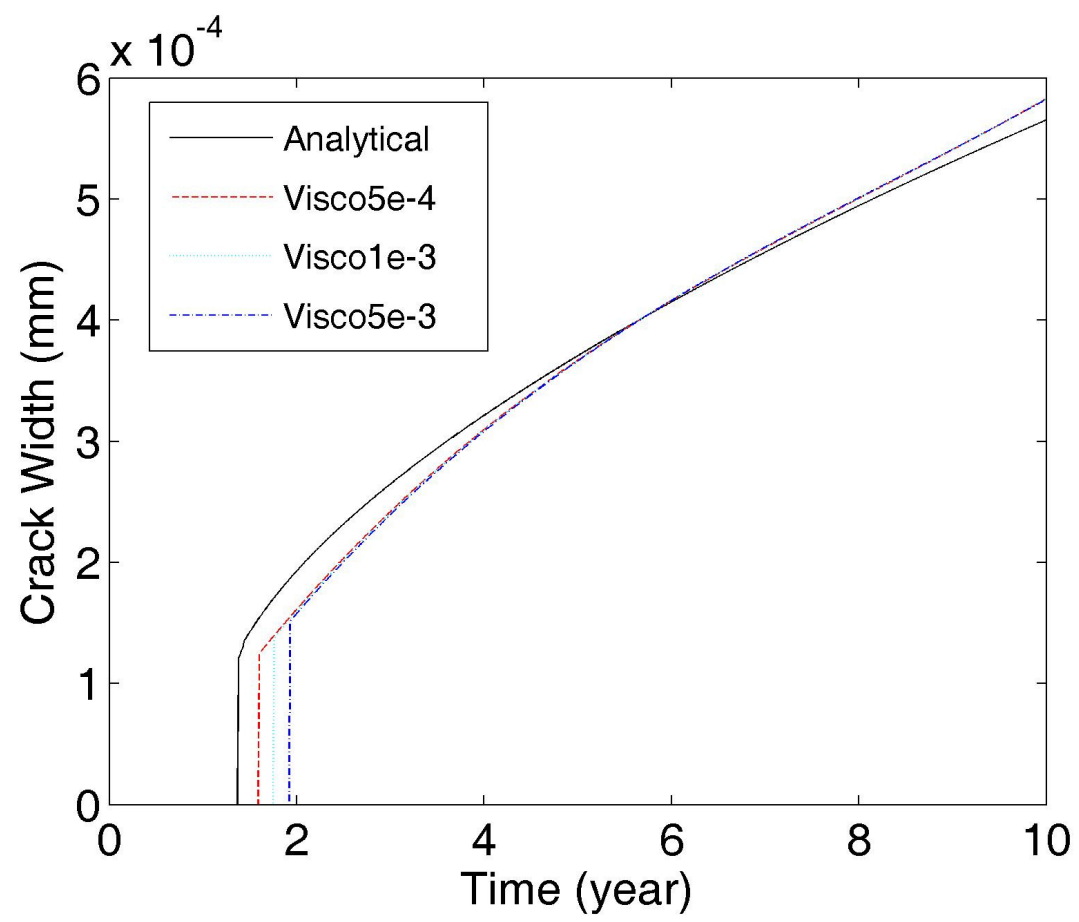

619 Time (year)

620 621

Figure 11 Effect of viscous regularization on the predicted concrete crack width 622 


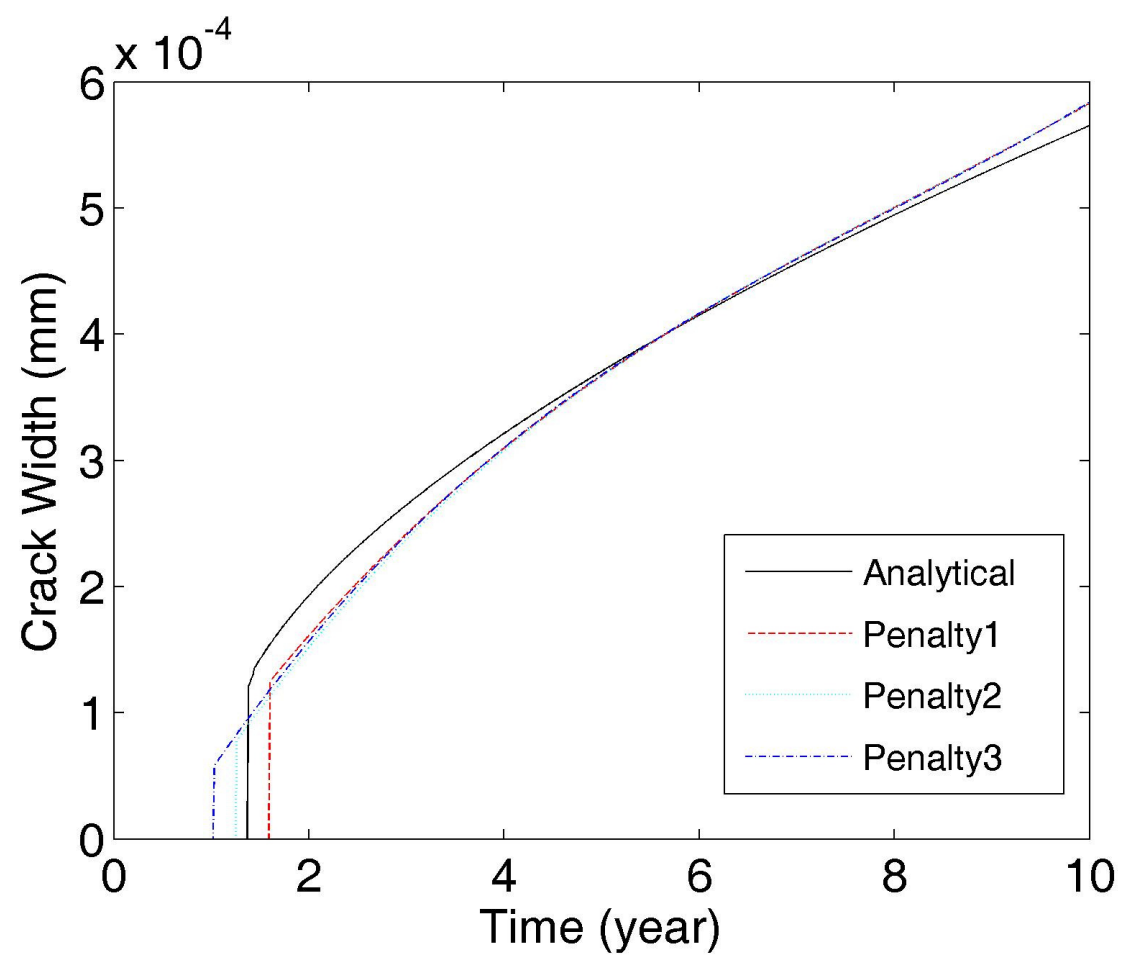

624 625

Figure 12 Effect of penalty stiffness on predicted concrete crack width 